\title{
Design of Intelligent Insect Monitoring System Using Deep Learning Techniques
}

\author{
V.Ceronmani Sharmila ${ }^{1}$, Neeraj Chauhan ${ }^{2}$, Rajdeep Kumar ${ }^{3}$, Suraj Kumar Barwal ${ }^{4}$ \\ \{csharmila@hindustanuniv.ac.in ${ }^{1}$, neerajchauhan098009@gmail.com² ${ }^{2}$ rajdeepkumar1109199 \\ 9@gmail.com ${ }^{3}$, barnwalsk14@gmail.com ${ }^{4}$ \} \\ ${ }^{1-4}$ Hindustan Institute of Technology and Science, No.1, Rajiv Gandhi Salai, OMR, Chennai-603103
}

\begin{abstract}
The agriculture field is growing up its potential to better the demand of food and delivers healthy and nutritious foodstuff. This project presents an insect or pest detection and classification for the plant using machine learning. It's a challenging part for the farmers to the crops which are acquiring defective and the degree or grade of excellence is also getting reduced day by day due to various pest or insect attacks. Earlier insect identification has been a big issue due to not well-skilled taxonomists to name the insects based on surface structure construction features accurately. Here, the proposed system will be a research tool for the study of early insects on the plants and leaves which will classify it using CNN and K-Means Clustering algorithm in machine learning. The detection analysis of insects was executed with shorter computational point in time for wang dataset using insect image Median Filter. The final classification results of accuracy were utilized to identify the pest and insects in the earliest period and increased the period to grow the harvest fertility and crop degree of excellence in the field of agriculture.
\end{abstract}

Keywords: Insect or pest classification algorithm, Insect or pest detection algorithm, Machine Learning, CNN algorithm, K-Means Clustering algorithm.

\section{Introduction}

Agriculture plays a vital role in everybody's life, as it is measured as the foundation of any country's economically system and come up with the growth of economically system development and determines the living criterion. The field of agriculture and processing food manufacture is a major sector between any countries. Growth of expanding is an important character for the sell abroad of good quality food production and agriculture. In progressing countries, the growth of economic earnings mainly depends on market demands and export supply chain earnings.

Insect attack is one of the biggest issues in the agriculture field part that degrades the quality of crops. Pest, Germ, Insect and weeds created a heavy damage to the harvest and result has reduces the degree of excellence for the last part of products. It requires high care for the pest and insect attack on crops which make effects in the field of growth crops. The pest and insect is the main cause behind the crop quality downfall and reduces crop productivity. Therefore, the monitoring of crops and evaluating the loss from insects is essential for ensuring quality of crops and making safety in the area of agriculture [1]. The computer perception of the machine is used in monitoring of soil and crops, plant leaf disease detection, fruit scaling, pest and insect acknowledgment and detection. Nowadays, many 
improvement are processed in the agriculture field, to distribute and determine the pests in stored grains using machine learning. Various different learning features methods are developed for spontaneous classification of area harvest insects [2].The main reason for studying to distribute and determine the pest and insects in rice, wheat and tea etc. in various plants applying machine learning techniques for insect and pest identification algorithms in the initial period of growth of crops. The classification of insects on a survey using image based insect recognition. The shape extraction features are used for insect and pest classification by using CNN models [3]. The insect and pest detection algorithm is very easy and useful in running point in time for detection of insects and pests in the area of agriculture. Image processing technique applies to segments for the foreground structure of insects and positioning of insects in the image using edge detection with a bounding box [4].

\section{Related Works}

The model is classification and detection of insects. Thenmozhi, et al. [5] designed a model as insect identification and detection in area harvest applying latest machine learning methodology. In this paper, we present the detection algorithm that consist of different dataset for insects such as Wang, Xie, Deng and IP102 for forefront extraction and outline identification to detect using highly complex background. The experiments have occurred for classification of 9 and 24 insect data of wang and xie dataset using structured characteristic using machine learning methodology such as KNN, SVM, ANN, NB and CNN model. The output of the CNN model proves that having greatest classification perfection is $91.5 \%$ and $90 \%$ for 9 and 24 data category of distinct insects or pest from different Wang and Xie dataset. The methods used for extracting the image as input insect dataset which will go under image pre-processing by applying filters with image augmentation technique for extracting the structured features of insects.

Chengjun et al. [6] designed a model for automatic identification of pests. In this paper, the classification methods perform on hand designed specifications such as HOG and SIFT in this work which gives the appearance of pest class in different poses. In this method, we are using unsupervised features learning methodology for unlabelled images for large amounts of image which transform the features of image obtained by low level features by alignment pooling (sparse coding). The experimental analysis on 40 insect species in the crop area that classify with multi-level learning specification which performs on the pest detection technique using the highest development methods in classification model. The representation of an image with multi-level learning specification consists of the feature encoding step which works with multi-level sampling step through unsupervised dictionary step.

Madhuri Devi et al. [7] designed a paper to Crop Insect Detection and Identification by KMeans. In this paper, the project scope is to classification and detection of the crop pest by providing the species type of pest and decreasing the use of fertilizer in the agriculture field. The images can be uploaded by farmer/user which is processed for extraction of image features. Colour images are based on image segmentation technique using the model as per Lab color space which will provide the k-means and EM clustering in fig 1. The region which is affected in the percentage form depends on various factors such as mean, entropy, RMS, energy, etc. are parameters which go under classification of the pest using the performance of multi SVM. 
Yong He et al [8] created an application in insect administration: an actual time method for identification of insects. Here, we approach a method to determine the pest based upon machine learning, which increases the mean average precision (map) and output will be enhanced by $9.7 \%$ with the compare of original model. It is a mobile application platform to get benefits for every farmer in the program, which helps to degrade the pest in real time which suggests agriculture for pest controlling in the plants. The application platform has increased his environmental adjustability, reaction speed and contrast by accuracy with earlier works and benefit of lower budget and easy functioning which effectively improves the agriculture production efficiency. We generally focus on three major architectures sectors for detection of objects: R-FCN, Faster R-CNN, and SSD and use a method to avoid overfitting as dropout.

Hari Shankar et al [9] develop an App for detecting the insect, herbs and disease identification using open computer perception, which gives much attention to continuous agriculture which is an essential area for monitoring the crops growth for the most nutritious way of growth. We are proposing a system which will be easy to handle the various multiple tasks at a cheap cost. We utilizes a technology in deep learning and ANN algorithm for UAV that helps a region to specify which are impacted with chemicals in that proper field, with complete structure price effectiveness. Using a central operating system to visualise agriculture using OpenCV, and easiest user interface for observing purposes.

In [10], the author proposed this method, where he has used some kind of trapping method using sticky materials. Pest will be attracted towards these traps with the help of some smelly material. Here insects get glued, this is an exhausting way to reduce pests, this method is not applicable on those pests which cannot fly, or those insects which are at initial level. Among many techniques to detect pests or insects, the author chooses clustering. Clustering works by basically breaking the image on the basis of multiple factors, like area, height, entropy, colours, geometries, distances etc. In this proposed method for detecting the objects from an image, authors have transformed an RGB image to another colour space. Subsequently, applying K-Mean Clustering which will identify different objects/factors. This technique was made to detect the affected areas/parts in the human body even from different diseases.

\section{Proposed System}

In the previous work, many machine learning methodologies has been utilised for pest classification and detection such as Artificial Neural Network (ANN), Naïve Bayes (NB), knearest neighbors, Support vector machines (SVM) and convolutional neural network (CNN) model. The highest classification accuracy of $90 \%$ was achieved for Wang dataset using the CNN model [5]. Here, we have introduced two different classification algorithms random forest (RF) and logistic regression (LR) for comparing and analysing the best accuracy result. This study puts a step ahead of a far-reaching model that would expedite the detection and classification of the insect/pests by using CNN [11] and K-mean clustering methods. In this research paper, the image has been segmented from insect dataset by using updated K-Mean clustering segmentation technique and $\mathrm{CNN}$ algorithm which will identify and label the pests or any other object from the image. The feature extraction will be done using GLCM and classified using CNN to classify pests [12]. Using the multi-class insect/pest identification 
using CNN architecture, High accuracy achieved which can be useful for identifying pests / insects in the agriculture field.

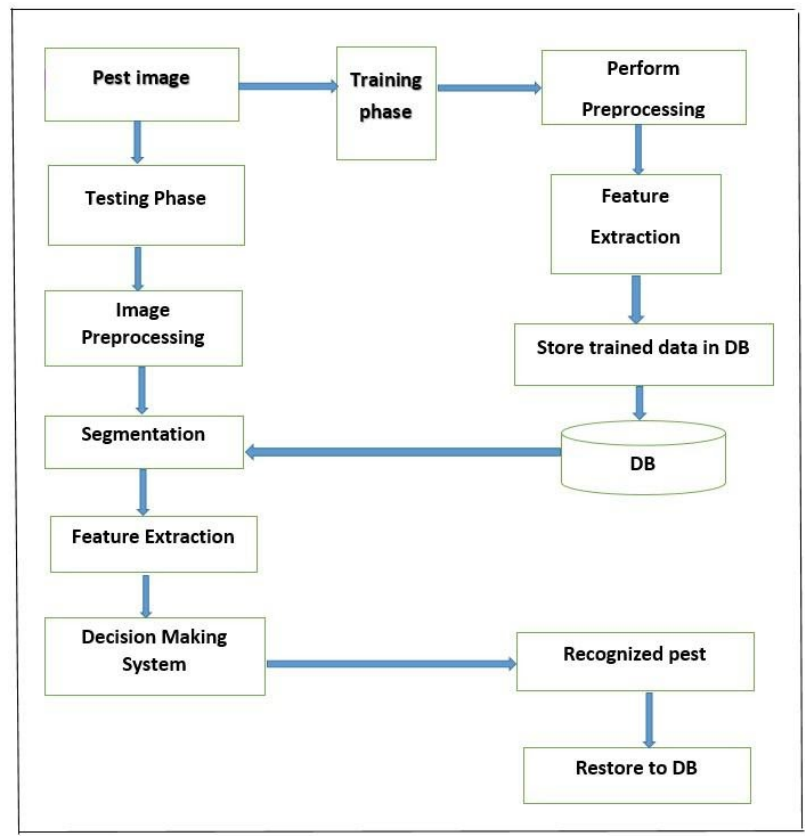

Fig.1. Functional Architecture

\section{Methodology}

\subsection{Dataset}

\section{A. Wang Dataset}

The insect and pest dataset repository consists of 9 classes and 282 total no of records of insect images attributes, but all the experiments refer to use a subset 2 or 3 attributes. The computational time for insect detection is less than $(<5)$ five $\min [5]$.

The list of 9 classes that are significant for the experiment-

i) Locusta migratoria

ii) Parasa lepida

iii) Gypsy moth larva

iv) Empoasca flavescens

v) Spodoptera exigua

vi) Chrysochus chinensis

vii) Laspeyresia pomonella larva

viii) Atractomorpha sinensis

ix) Laspeyresia pomonella

\subsection{Image Pre-Processing}

Image pre-processing performs major tasks such as resized image, adding noise, filtering, HSV, Grey image, Binary image, complement image, edge detection segmentation, dilated image. 


\subsection{Shape Feature Extraction}

In image processing, the feature extraction begins with a preliminary position of calculated values and construct into another values (features) considered to be communicative and non-significant, facilitating the consequent studying and normalization steps, and in few instance dominating to enhanced human interpretations [13]. Feature extraction is connected to proportional decrease.

\subsection{Insect Classification}

The pests are categorized within different classes applying the seven machine learning methods such as ANN, RANDOM FOREST , SVM, KNN, , LOGISTIC REGRESSION, NB and $\mathrm{CNN}$ classifier which are defined as follows[14].

1. ANN classifier -An easy neural network has an input, unseen, and output layer with linkage. At first, randomly weights are allocated. The last linkage weights were determined, and the activation rate of the output layer was measured. A feed-forward multi-layer artificial neural network is sketched to accordingly detect and divide mature-stage whiteflies and thrips in greenhouses [15]. The ability of the system of pest recognition system was developed by applying an ANN model to identify beet foreclosures (Spodoptera exigua) of different species of family in the fields.

2. SVM classifier -It is a supervised machine learning algorithm. Here, the measurement space build on the different types of characteristics that we are having. The values item is plotted as a point in a $\mathrm{k}$-dimensional space, where we have ' $\mathrm{k}$ ' attributes with the data of a specific coordinate, presenting the greater point. Pest classification is done by recognizing the hyper-plane, which separates the orbits into excellently. SVM [16] was applied completely to reduce the intersection error rate completely for the identification of mosquito and fruit flies [17].

3. KNN classifier -It is an idle learning algorithm that doesn't implement other specification. Here, weight is credited to the contribution of neighbors, like that adjacent neighbors always provide extra to the average than neighbors. In transforming identification difficulty with great amplitude and less samples, the KNN algorithm obtains an enhanced detection class for butterfly species [18].

4. Naive Bayes -It is formed on the probability of the Bayes theorem and take it an independence between every prophets. This classifier only observe the appropriation that the insect for a specific characteristics is not connected to the existence of over another attributes in the category. The Naive Bayesian classification was used to conclude a pests category probabilities that a given pair from a grain harvest pests dataset is a specific insect or pest category [19].

5. Random forest classifier-It is a classification algorithm that consists of multiple decision trees. It utilizes to captures and randomness of features when constructing every particular tree, trying to generate an unrelated forest area of trees whose prediction by the committee is increased accurate than that of particular specific tree. Random forest [20] constructs several decision trees and combines them all together to obtain a high accurate and secure prediction. Random forests have almost the similar hyper parameters as the decision tree or bagging classifier. Growing trees, random forest sum up additional unexpected to the system. 
6. Logistic Regression-Logistic regression is firstly a supervised classification algorithm. In a classification complication, the goal variable (or yield), $y$, can take only distinct data for a prescribed feature (or input), x. Contrary to famous faith, logistic regression is a regression model. Regression is a classification algorithm used for observing discrete sets of category. Logistic regression converts its output applying the logistic sigmoid function to give back probability values.

7. CNN classifier-The term "conventional neural network" suggests that the network employs a mathematical operation called convolution. Sensory networks are a special class of neural networks used for the characterization of at least one of their layers in place of normal matrix multiplication [21].

The CNN layer is divided into further types of layers are mentioned as-

1. Input Layer: Input of an image with height, width and depth.

2. Convolution Layer: The output is produced using dot product between image patch and all filters.

3. Activation Function Layer: Convection layer containing the elevated activation function of the output. Ex- RELU

4. Pool Layer: Two types of Common pooling layers are average pooling and maximum pooling.

5. Fully Connected Layer: This step takes input from the earlier steps and generates a class score and output.

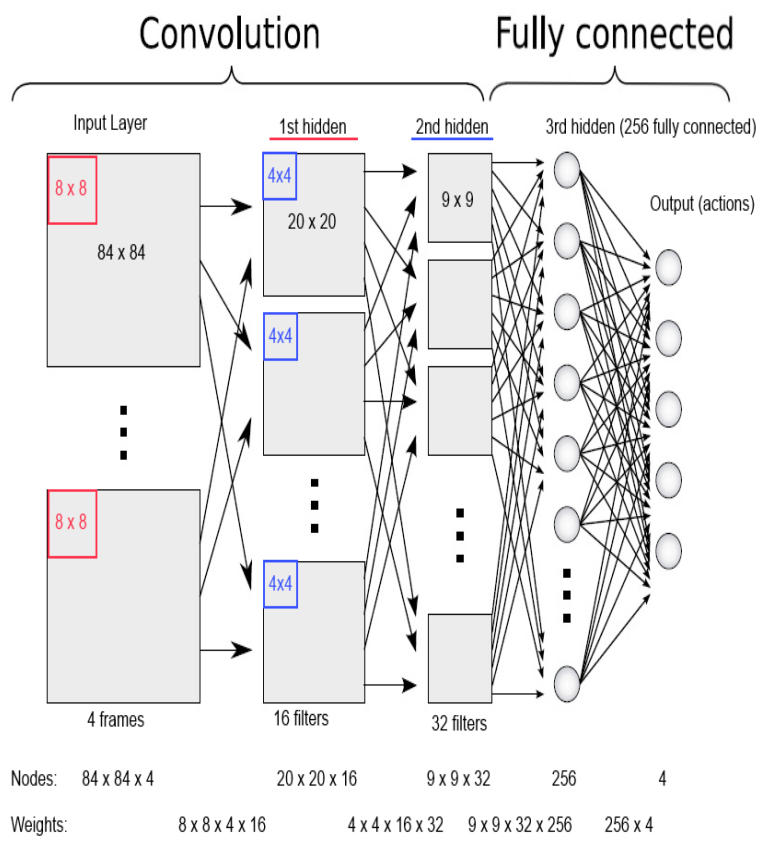

\subsection{Pest Classification Results}

Fig.2. CNN Layered Architecture

The classifier performance applying shape characteristics derived from image processing techniques with machine learning techniques which includes SVM, KNN, Nave Base, ANN, and CNN [22], for comparing the classification accuracy with every methodology of model in insect classification. Are also used. A nine folder cross checking was used to the Wang dataset 
for 1359 insect and pest images. Comparing of the various methodology was performed just after the solution were composed in fig. 3 shows the results were achieved for insect and pest classification for Wang applying different machine learning algorithms techniques.

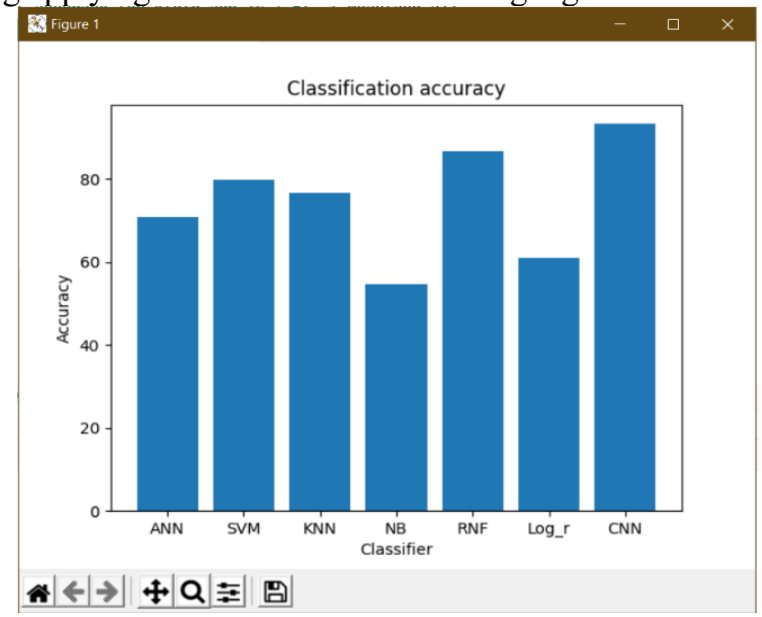

Fig.3. Accuracy Graph of different Classifiers

Different classifiers in size extracted from 9 insect classes of the WAND dataset have been applied. The classification accuracy is $86.9 \%$ and $70.8 \%$ for the 9 classes of pests obtained with nine size characteristics is predicted by Random Forest and ANN, respectively. The Gaussian naive Bayes classifier and logistic regression results resulted in lower accuracy of $54.54 \%$ and $60.92 \%$ due to the feature's dependence, and it weighs all features equally. As the writer applied moment-invariant characteristics with SVM classifiers and achieved an accuracy of $79.95 \%$ for 9 insect and pest classes. In KNN, 10 and the number of neighbors chosen as the Euclidean distance metric are used so it can give an accuracy of $76.43 \%$. The CNN model has yielded $93.9 \%$ accuracy for 9 insect and pest classes as it relates to high discriminating characteristics in insect or pest images.

Time to process 9 class of insect datasets in different classifier system. The calculation time for different algorithm are ANN, SVM, KNN, Naive Bayes, Random Forest and logistic regression was shorter than the proposed $\mathrm{CNN}$.

The order of time consumption is $\mathrm{NB}<\mathrm{KNN}<\mathrm{SVM}<\mathrm{RNF}<\mathrm{LG}<\mathrm{ANN}<\mathrm{CNN}$.

\section{Insect Pest Identification}

\subsection{Image Pre-Processing}

Image pre-processing performs major tasks Such as resized image, adding noise, filtering, HSV, Grey image, Binary image, complement image, edge detection segmentation, dilated image [23].

The matlab is a software which recognises the original image of an insect and performs the various operations for pre-processing of an image. During pre-processing of an image it undergoes image resizing which resizes the original image with less resolution [24]. When the image is resized then noise is added to filter the image with high accuracy. After filtering it, HSV is applied and simultaneously converted into Gray image. 
We applied binary conversion to convert the image into black and white and similarly we applied complement image to do vice versa of black and white image. Finally, the image undergoes an edge detection segmentation process for detecting the edge of the image of the insect.

In this methodology, we applied median filter to find the mse and psnr using noise added image [25].

Mean Square Error -

$[$ row, col] $=\operatorname{size}(\mathrm{I})$;

$\mathrm{mse}=\operatorname{sum}\left(\operatorname{sum}\left((\mathrm{I}(1,1)-\mathrm{K}(1,1)) .^{\wedge} 2\right)\right) /($ row $* \operatorname{col})$;

Peak Signal to Noise Ratio -

$\mathrm{psnr}=10 * \log 10(255 * 255 / \mathrm{mse})$;

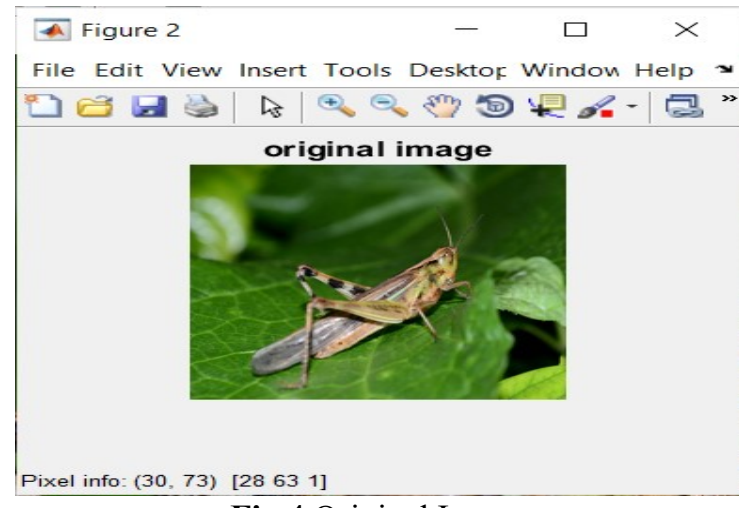

Fig.4.Original Image

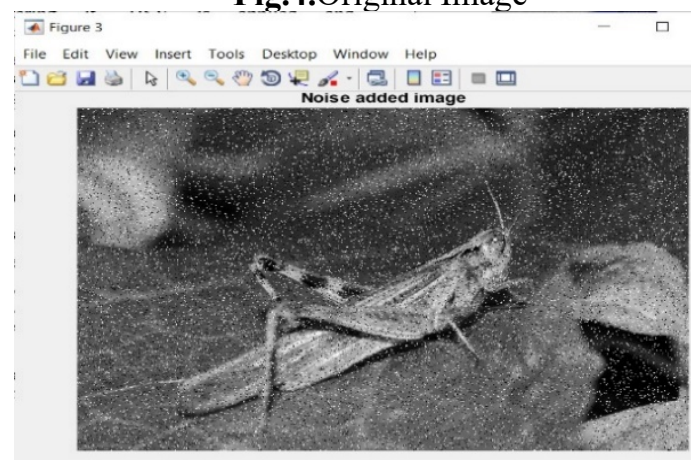

Fig.5. Noise Added Image 


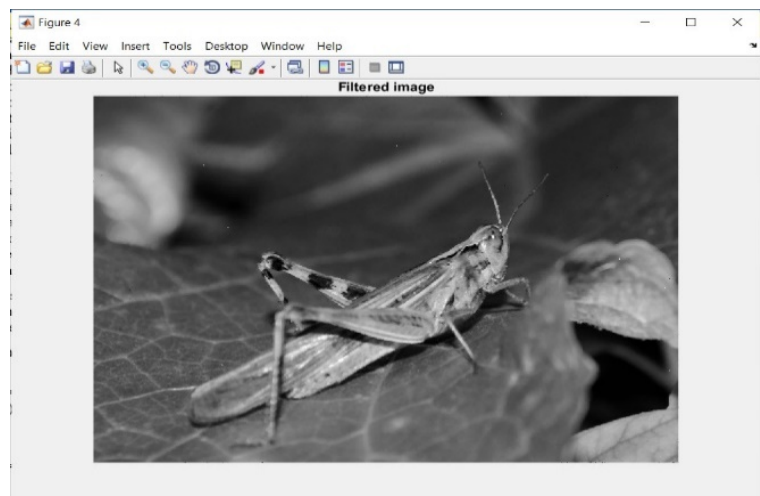

Fig.6.Filtered Image

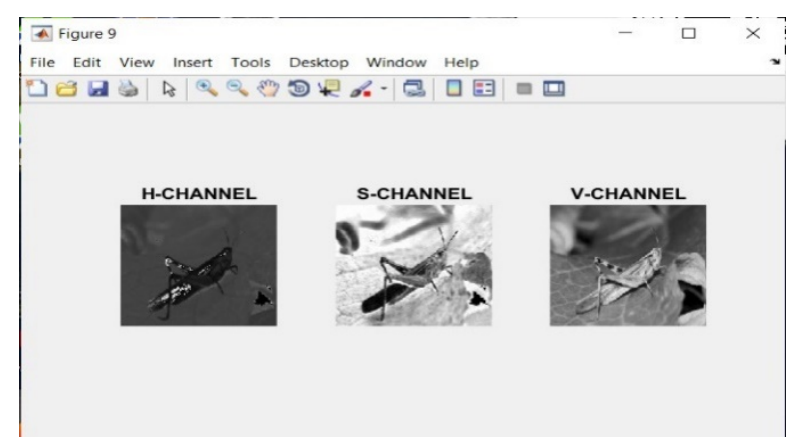

Fig.7. HSV Image

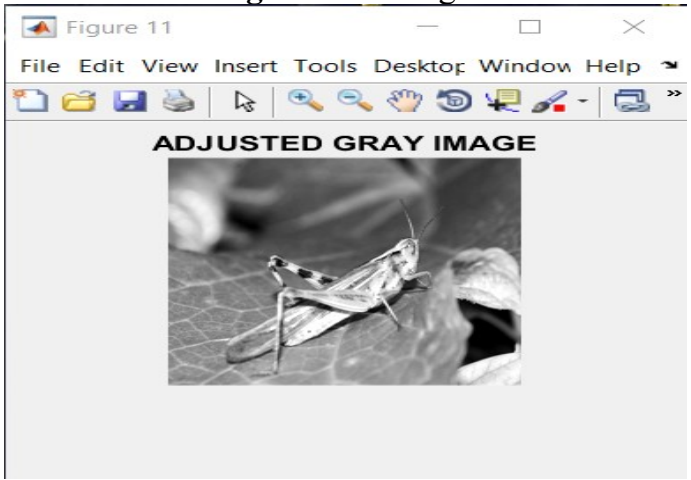

Fig.8. Adjusted Gray Image 


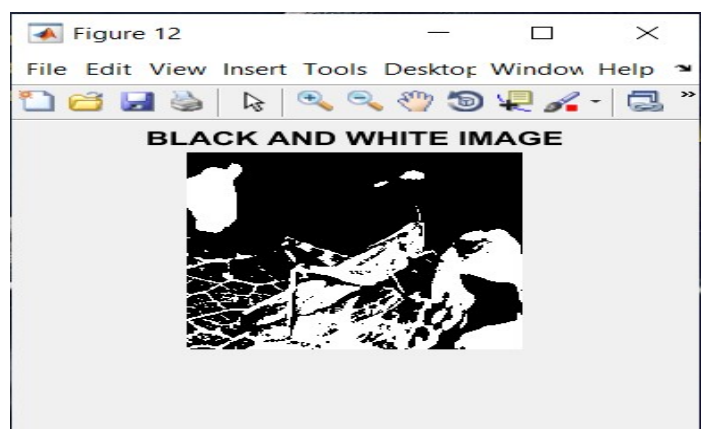

Fig.9. Binary Image

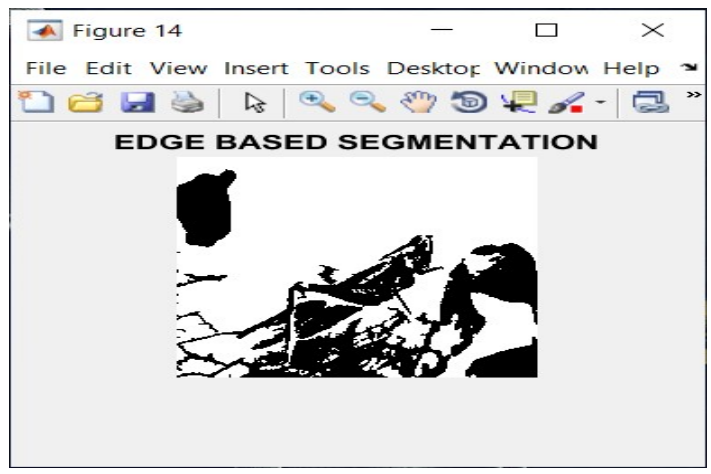

Fig.10.Edge Based Image

From this, we can pre-process the image using various operations on it for performing different tasks in the image. Hence we can find the mse and psnr from the above.

\subsection{Segmentation}

In computer perception, image segmentation is the procedure for splitting an image into multiple segments. The aim for segmenting an image is to replace the description of an image into different which is higher significant and simple to inspect. It is usually applied for identifying objects and generating boundaries.

In this the input image is segmented based on the color bands of the lab $\mathrm{a}^{*} \mathrm{~b}^{*}$. The segmentation will be achieved by using the k-means clustering at $t$ multiple level. The cluster is nothing but the group of the pixel belonging to the same color bands.

\subsubsection{Clustering methods}

The K-means algorithm is an iterative method that is applied for splitting an image into $\mathrm{K}$ clusters [26]. The basic algorithm is

- Select K cluster center, either randomly or based upon some heuristic technique, for example K-means++

- Provide every pixel in the image to the cluster that decreases the distance among the pixel and the cluster center.

- Compute the cluster centers by making average of the every pixels in the cluster.

- Repeat steps 2 and 3 until convergence is acquired (i.e. no pixels change clusters)

Enhanced Images are classified into three different phenomena to detect the best enhanced images from them to detect the best one from them. 
Here, we made the enhanced images as enhanced1, enhanced2, enhanced3 which undergoes detection of images in segmentation process method [27].

Segmentation images first show the segmentation of the image from the enhanced image which turns into a completely segmented image which is the next peak level of segmentation.

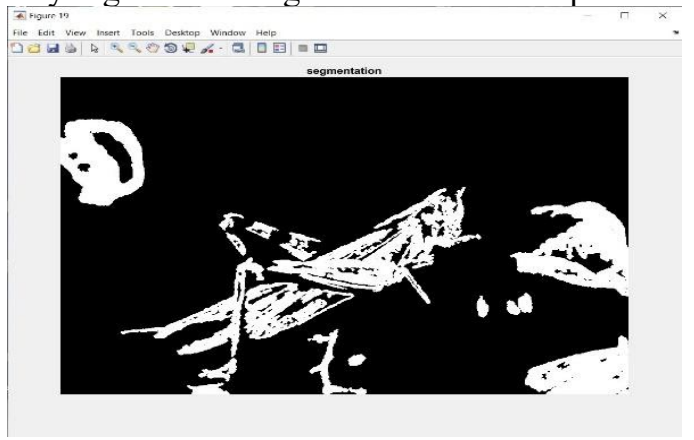

Fig.11. Pre Segmented Image

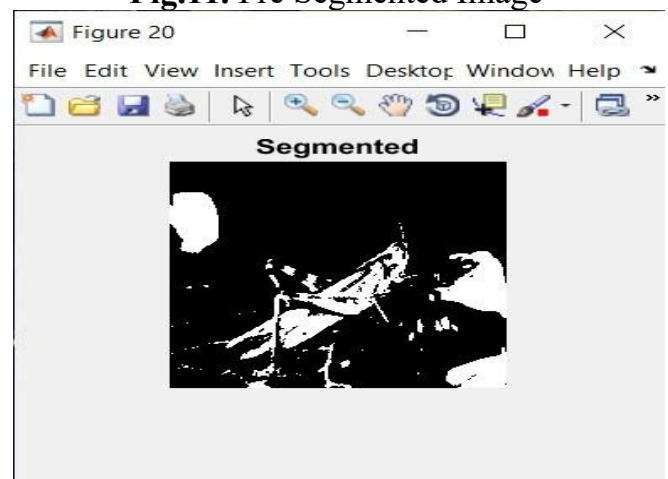

Fig.12.Segmented Image

\subsection{Shape Feature Extraction}

In image processing, feature extraction begins with a starting position of calculated values and construct into inherit values considered to be communicative and non-significant, facilitating the subsequent studying and normalization steps, and in few instance dominating for enhanced human clarification. Feature extraction is connected to proportional for decrease.

When the input of the values to an algorithm is too big to be procedure and it is doubtful to be extreme, then it can be transformed into a limited range of characteristics.

The picked characteristics are supposed to hold the particular information from the input values, so that the expected work can be transformed by applying this decrease presentation in place of the complete initial values [29].

Features are extracted using GLCM matrix such as:-

correlation, contrast, energy, entropy, inverse different moment, homogeneity, smoothness, mean, standard deviation, variance, skewness, RMS.

\subsection{Classification and Labelling}

$\mathrm{CNN}$ is a kind of deep, feed-forward artificial neural networks (where relations among nodes do not form a cycle) \& utilize a difference of multilayer perceptron measured to require minimum pre-processing. These are motivated by animal visual cortex. 
Training is determined using a "labelled" dataset of inputs in a wide group of representative input patterns that are attached with output response. Training uses generalpurpose methodology to recursively determine the weights for middle and final feature neurons.

Again applying the standard neural network which would be equal to a CNN, because the unit of factors will be seriously greater, the training period will also be increment simultaneously. In a $\mathrm{CNN}$, since the unit of factors is extremely decreasing, training period is simultaneously reducing [30]. Also, supposing excellent training, we can make a standard neural network whose characteristics will be similar to a CNN. But in experimental training, a standard neural network equally to $\mathrm{CNN}$ will have the higher characteristic, which would be conduct to noise addition during the training procedure. Hence, characteristics of a standard neural network equally to a CNN will all the time be lower [31].

\section{Pseudo Code of the algorithm}

- Precondition: train, validate_dir = train/validate('filepath', parameters)

- Define input shape, no.of classes \& conv_size

- $\quad$ model $=$ sequential model

- model.add(Conv2d(attributes.....) x 3)

- $\operatorname{model} . \operatorname{add}(\max$ pooling layer(attributes......) x 3 )

- $\quad$ model.add (flatten())

- model.add(dense(512 neurons with relu activation))

- $\quad$ add another dense layer but with softmax activation

- $\quad$ perform model.compile(loss, metrics and optimizer attributes)

- model.fit(specify train dataset, no.of epochs, steps per epoch, specify validation dataset).

- $\quad$ visualize accuracy and performance metrics as done previously

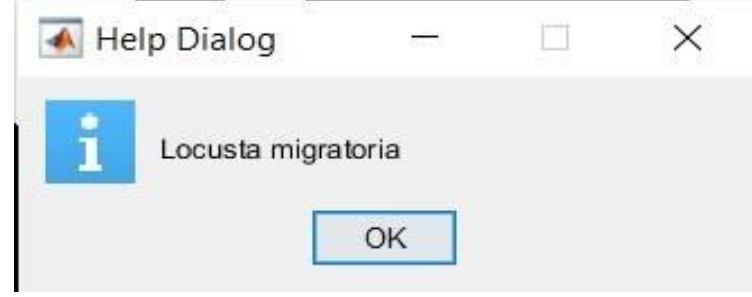

Fig.13. Labelled Image

\section{Conclusion}

In this research paper, we have compared the classification accuracy of CNN, ANN, SVM, KNN, Naïve Bayes, Random Forest and Logistic regression. In the proposed system, we proposed a pest detection by using a feature set which is executed on the dataset as a training, in which the characteristic values are generated and stored in the system database. User's input selected image is proceed to the system to extract input pest image characteristics. The image is then processed in multiple steps by using region-based segmentation of the images by applying the K-means clustering to generate the features of the dataset to give the final result by using the help of CNN to classify the pest and insect. 


\section{References}

[1] Al-Hiary, H., S. Bani-Ahmad, M. Reyalat, M. Braik, and Z. AlRahamneh. (2011). "Fast and accurate detection and classification of plant diseases". International Journal of Computer Applications, 17(1): 31-38. Doi:10.5120/2183-2754

[2] C. Xie, R. Wang, J. Zhang, P. Chen, W. Dong, R. Li, T. Chen, H. Chen (2018) "Multi-level learning features for automatic classification of field crop pests", Computer Electron Agriculture, 152, pp. 233-241. Doi: 10.1016/j.compag.2018.07.014

[3] M. Martineau, D. Conte, R. Raveaux, I. Arnault, D. Munier, G. Venturini (2017). "A survey on image-based insect classification Pattern Recognition”, 65 pp. 273-284. Doi:/10.3390/insects11080458

[4] S.G.Gino Sophia, V. Ceronmani Sharmila, "Zadeh max-min composition fuzzy rule for dominated pixel values in iris localization", Soft Computing, Springer Journal, November 2018, Vol. 23, Issue-6, ISSN: 1432-7643, 1873-1889.

[5] K. Thenmohiz, U. Srinivasulu Reddy. (2017)" Image processing techniques for insect shape detection in field crops," IEEE pp. 978-1-5386-4031-9. Doi: 10.1109/ICICI.2017.8365226

[6] Chengjun Xie, Rujing Wang, Jie Zhang, Peng Chen, Wei Dong, Rui Li, Tianjiao Chen, Hongbo Chen. (2018). "Multi-level learning features for automatic classification of field crop pests", ScienceDirect, 152 pp.233-241. Doi: 10.1016/j.compag.2018.07.014

[7] Madhuri Devi Chobey, Humera Tamkeen (2019)."Crop Pest Detection and Classification by K-Means and EM Clustering". International Research Journal of Engineering and Technology (IRJET) Volume: 06.

[8] Yong He, Hong Zeng, Yangyang Fan, Shuai Zheng Ji, Jianjian Wu. (2019). “Application of Deep Learning in Integrated Pest Management: A Real-Time System for Detection and Diagnosis of Oilseed Rape Pests", Mobile Information Systems. Doi:10.1155/2019/4570808

[9] Hari Shankar RL, A. K. Veeraraghavan, Uvais, K. Sivaraman, S. Shreyas Ramachandran. (2018)."Application of UAV for pest, weeds and disease detection using open computer vision", IEEE Xplore Part Number: CFP18P17-ART; Doi: 10.1109/ICSSIT.2018.8748404

[10] Sushma R Huddar, Swarna Gauri, (2018). "Novel algorithm for segmentation and automatic identification of pests on plants using image processing," IEEE 2018.

[11] L.Q. Zhu, M.Y. Ma, Z. Zhang, P.Y. Zhang, W. Wu, D.D. Wang, et al. (2017). "Hybrid deep learning for automated lepidopteran insect image classification" Orient Insects, 51, pp. 79-91. Doi:10.1080/00305316.2016.1252805

[12] Argenti, F., L. Alparone, and G. Benelli. 1990. "Fast algorithms for texture analysis using cooccurrence matrices." IEEE proceedings, 137, (6): 443-448 Doi: 10.1049/ip-f-2.1990.0064

[13] S.N. Yaakob, L. Jain, (2012). "An insect classification analysis based on shape features using quality threshold ARTMAP and moment invariant" Appl Intell, 37 (1) pp. 12-30. Doi:10.1007/s10489-011-0310-3

[14] Preetha Rajan, Radhakrishnan. B. (2016).” Detection and Classification of Pests from Crop Images Using Support Vector Machine". ICETT, IEEE. Doi: 10.1109/ICETT.2016.7873750

[15] J. Wang, C. Lin, L. Ji, A. Liang "A new automatic identification system of insect images at the order level” (2012). Knowl-Based Syst, 33 (2012), pp. 102-110. Doi: $10.1016 /$ j.knosys.2012.03.014

[16] Chih-Wei, H., and C. Lin. (2002)." A comparison of methods for multiclass support vector machines." IEEE Transactions on Neural Networks, 13(2): 415-425. Doi: 10.1109/72.991427

[17] M. Fuchida, T. Pathmakumar, R.E. Mohan, N. Tan, A. Nakamura, (2017)"Vision-based perception and classification of mosquitoes using support vector machine" Appl Sci., 7 p. 51. Doi: 10.3390/app7010051

[18] Z. Iqbal et al.," An automated detection and classification of citrus plant diseases using image processing techniques: A review," (2018). Computers and Electronics in Agriculture $153 \mathrm{pp}$. 12-32. Doi: 10.1016/j.compag.2018.07.032.

[19] Sunil Joy Dommati, Ruchi Agrawal, Ram Mohana Reddy G, Sowmya Kamath S. (2012) "Bug Classification: Feature Extraction and Comparison of Event Model using Naive Bayes Approach." ICRTCIE (2012). 
[20] Junfeng Gao, David Nuyttens, (2018) "Recognizing weeds in a maize crop using a randomforest machine-learning algorithm and near-infrared snapshot mosaic hyper spectral imagery," IAgrE. Published by Elsevier Ltd.

[21] Gino Sophia, Ceronmani Sharmila, "Computer vision algorithms for dominant contact lens feature extraction using fuzzy-logic-based classifications", Soft Computing, Vol. No. 24, Issue No. 18, ISSN : 1432-7643, pp. 14235-14249, March 2020.

[22] Y. Shen, H. Zhou, J. Li, F. Jian, D.S. Jayas. (2018). "Detection of stored-grain insects using deep learning", Computer Electron Agriculture, 145, pp. 319-325. doi:/10.1016/tii 2017.11.039

[23] C. Wen, D. Guyer ,(2012)."Image-based orchard insect automated identification and classification method" Comput Electron Agric, 89, pp. 110-115. Doi: 10.1016/j.compag.2012.08.008

[24] S. G. Gino Sophia, V. Ceronmani Sharmila. (2018) "Morphological-Based Localization of an Iris Image", Advances in Intelligent Systems and Computing, Springer,ISBN: 978-981-13$1579-4$.

[25] A.M. Hambal, Z. Pei, F.L. Ishabailu (2017), "Image noise reduction and filtering techniques" Int J Sci Res, 6.pp. 2033-2038

[26] Yogesh Kumar, Ashwini Kumar Dubey, (2017)."Pest detection using adaptive thresholding," IEEE. Doi: 10.1109/CCAA.2017.8229828

[27] M.C. Bakkay, S. Chambon, H.A. Rashwan, C. Lubat, S. Barsotti. (2017)."Automatic detection of individual and touching moths from trap images by combining contour-based and regionbased segmentation". IET Comput Vis, 12, pp. 138-145. Doi: 10.3390/agriculture10050170

[28] Yu X, Zhuorui S, Seishi N. (2002). "Measuring geometrical features of insect specimens using image analysis." In: Conference on Asian federation information in agriculture (AFITA).

[29] Nivetha. S, Ceronmani Sharmila. V. (2017). "Implementation of Connected Dominating Set in Social Networks using Mention Anomaly", Artificial Intelligence and Evolutionary Computations in Engineering Systems, Springer, pp. 777-790.

[30] Gino Sophia, Ceronmani Sharmila. (2020) "Water management using genetic algorithm-based machine learning", Soft Computing, Vol. No. 24, Issue No. 22, pp. 17153-17165.

[31] L. Deng, Z. Wang, C. Wang, Y. He, T. Huang, Y. Dong, et al. (2020), "Application of agricultural insect pest detection and control map based on image processing analysis" J Intell Fuzzy Syst, 38 pp. 379-389. Doi: 10.1016/j.inpa.2020.09.006 